\title{
An automated approach for analysis of Fourier Transform Infrared (FTIR) spectra of edible oils
}

\author{
Siong Fong Sim*, Woei Ting \\ Universiti Malaysia Sarawak, Faculty of Resource Science and Technology, 94300 Kota Samarahan, Sarawak, Malaysia
}

\section{A R T I C L E I N F O}

\section{Article history:}

Received 9 September 2011

Received in revised form

10 November 2011

Accepted 10 November 2011

Available online 17 November 2011

Keywords:

Fourier Transform Infrared

Peak detection

Peak assignment

Peak matching

Edible oils

\begin{abstract}
A B S T R A C T
This paper reports a computational approach for analysis of FTIR spectra where peaks are detected, assigned and matched across samples to produce a peak table with rows corresponding to samples and columns to variables. The algorithm is applied on a dataset of 103 spectra of a broad range of edible oils for exploratory analysis and variable selection using Self Organising Maps (SOMs) and $t$-statistics, respectively. Analysis on the resultant peak table allows the underlying patterns and the discriminatory variables to be revealed. The algorithm is user-friendly; it involves a minimal number of tunable parameters and would be useful for analysis of a large and complicated FTIR dataset.
\end{abstract}

(C) 2011 Elsevier B.V. All rights reserved.

\section{Introduction}

Fourier Transform Infrared (FTIR) is a form of vibrational spectroscopy where infrared radiation is passed through a sample resulting in absorption of the radiation that stimulates vibrational motions. Fundamentally, a molecule is represented by a set of flexible and moving atoms where the atoms constantly oscillate around average positions. When the vibration of the atoms produces an oscillating electric field same as the frequency of the IR radiation, it gives rise to peaks (often referred to as bands) in vibrational spectrum. Each spectral peak is characterised by its frequencies and amplitude as a molecule only absorbs at frequencies corresponding to its molecular modes of vibration in the infrared region [1].

FTIR has been widely used as a routine diagnostic tool for qualitative and quantitative analyses. The major advantages of FTIR over other analytical methods are it is relatively fast, non-destructive and cost effective; in addition, only a small amount of sample is required [2]. FTIR in combination with various multivariate data analysis methods have allowed rapid evaluation of a large volume of spectral data for quality control [3], quantification [4] and pattern recognition [5-7]. Typically, this involves the profile of the entire spectrum or specific spectral regions containing relevant information [8]. In this paper, we report an automated computational approach for analysis of FTIR spectra and demonstrate the

\footnotetext{
* Corresponding author. Tel.: +60 82 582995; fax: +60 82583160.

E-mail addresses: sfsim@frst.unimas.my, siongfong@gmail.com (S.F. Sim).
}

algorithm for the analysis of edible oils. Fundamentally, the algorithm finds peaks present in each sample and matches the peaks across samples to produce a peak table with rows corresponding to samples and columns to variables for multivariate data analyses. The algorithm is also designed to assign peaks according to frequencies suggesting the possible functional groups. This would speed up the peak assignment process which is often performed manually and serves as a guide for non-expert users in spectra interpretation. In fact, many automated data processing algorithms have been developed for various applications, i.e., GC-MS [9], GC-DMS [10], GC-GC [11], LC-MS [12], NMR [13] and HPLC [14]. They have been used for microbial, metabolomics and food study [15-20]. The method is particularly well-accepted when the data processing software available with the workstation can no longer cater the demand of the analyst, i.e., he/she wishes to look at hundreds of peaks in a complex matrix and compare the peaks across multiple samples. Hence, the algorithm offers an alternative to analyse large high dimensional FTIR datasets when interpreting complicated superimposed spectra becomes infeasible and peak integration becomes tedious and time consuming.

\section{Materials and methods}

\subsection{Dataset}

This dataset consists of 103 FTIR spectra of 8 types and 16 brands of edible oils. The samples represent a broad range of edible oils from olein palm oil, blended palm oil (consists of olein palm oil, 